\title{
Synergistic Physician and Patient Behavioral Interventions as Management Strategy for Effective Population-Based Hypertension Control
}

\author{
Pei-an Betty Shih • Daniel T. O'Connor
}

Published online: 25 April 2010

(C) The Author(s) 2010. This article is published with open access at Springerlink.com

Svetkey LP, Pollak KI, Yancy WS Jr., et al.: Hypertension Improvement Project: randomized trial of quality improvement for physicians and lifestyle modification for patients. Hypertension 2009, 54:1226-1233.

\section{Rating}

-Of importance.

\section{Introduction}

Effective hypertension management is still a clinical challenge. The prevalence of hypertension continues to rise in the western world, despite awareness of its serious cardiovascular consequences [1]. Adequate blood pressure (BP) control is of clear public health importance, but recent reports indicate that as many as two thirds of those being treated for hypertension in the United States do not have BP under proper control [2]. To address this important public health issue, a large study evaluating the efficacy of a nonconventional, two-level (patient and physician) management strategy for hypertension management was timely.

P.-a. B. Shih $(\bowtie) \cdot$ D. T. O'Connor

Department of Medicine, University of California, San Diego, Center for Human Genetics and Genomics,

Pharmaceutical Sciences Building (PSB), Room 4261, 9500 Gilman Drive,

La Jolla, CA 92093, USA

e-mail: pbshih@ucsd.edu

\begin{abstract}
Aims
Using a large, prospective, and randomized communitybased trial, the Hypertension Improvement Project (HIP) recently reported their findings on the comparative effectiveness of 1) physician intervention, 2) patient intervention, or 3) the combination of such interventions [3].
\end{abstract}

\section{Methods}

Published in the journal Hypertension in November 2009, the HIP study demonstrated differences in BP-lowering effects between patient intervention, physician intervention, and combined interventions. The patient-intervention strategy consisted of 20 weekly group sessions over 6 months, conducted by experienced behavioral personnel. The focus of the intervention was the use of motivational interviewing techniques to assist patients toward weight loss if overweight, to increase awareness of dietary approaches to controlling BP (with the Dietary Approaches to Stop Hypertension [DASH] dietary pattern [4]), to increase moderate-to-vigorous physical activity, and to reduce sodium intake and moderate alcohol intake. Additionally, the patient intervention aimed to encourage adherence to the antihypertensive medication regimen. In short, this patient-intervention regimen was designed to "promote frequent self-monitoring, feedback, goal setting, and social support and used motivational interviewing techniques" [3].

The physician intervention strategy consisted of two training modules for the clinicians: one addressed the Seventh Joint National Committee on Prevention, Detection, Evaluation and Treatment of High Blood Pressure (JNC-7) guidelines [5], and the second addressed lifestyle modification for BP control. Additionally, each physician in 
this group received a pocket-sized laminated card that summarized the major JNC-7 guidelines, including lifestyle guidelines and a decision tree. Results of a quality improvement procedure assessing clinical performance measures and quarterly feedback on adherence to JNC-7 guidelines were provided to the physician intervention group. The goal of the physician-intervention regimen was to increase physicians' awareness of current recommendations for hypertension treatment goals and management.

The HIP study then evaluated the effectiveness between groups exposed to patient intervention alone, physician intervention alone, or both interventions combined, as compared with no intervention (usual care). To assess both shortterm and long-term BP consequences of these interventions, the HIP study reported systolic BP (SBP) and diastolic BP (DBP) changes at 6 and 18 months postrandomization.

\section{Results}

At 6 months, the patient-intervention group showed a significant decrease in SBP of $7.1 \pm 12.1 \mathrm{~mm} \mathrm{Hg}$ (mean \pm $\mathrm{SD} ; P<0.05$ ), with a net reduction (main effect adjusted for baseline pressure) of $2.6 \mathrm{~mm} \mathrm{Hg}(95 \% \mathrm{CI},-4.4$ to $-0.7 ; P=$ 0.01 ). The significant reduction of BP at 6 months in the patient-intervention group is perhaps not surprising, as patients are their best advocate for health and well-being. The support group and lifestyle-based interventions provided may have directly increased patients' motivation to lower their BP via tools taught to them such as better adherence to lifestyle modifications (healthier diet and increased physical activity), and through increased compliance with pharmacologic treatments. Additionally, patients who become more aware of their hypertension status are more likely to proactively communicate their BP concerns with their physicians, which in turn may help their physicians to make timely treatment recommendations.

On the other hand, patients in the group with the physician intervention only did not show a statistically significant drop in BP at the 6-month point; they had a mean decrease in SBP of $5.3 \pm 12.1 \mathrm{~mm} \mathrm{Hg}(P>0.05)$, but a net reduction (main effect adjusted for baseline pressure) of only $0.3 \mathrm{~mm} \mathrm{Hg}(95 \% \mathrm{CI},-1.5-2.2 ; P=0.76)$.

Svetkey et al. [3] demonstrated that the most effective outcome was seen in the group that received both patient and physician interventions. In this combined-intervention group, the SBP main effect (adjusted for baseline pressure) fell the most, at $9.7 \pm 12.7 \mathrm{~mm} \mathrm{Hg}(P=0.0072$, compared with all other groups). The data showed evidence of significant interaction between physician intervention and patient intervention $(P=0.03)$, suggesting that the effect of patient intervention was enhanced by coincident exposure to physician intervention (or vice versa).

\section{Discussion}

The results at 6 months suggest that physician intervention, which as a management strategy alone did not show significant benefit, may have enhanced the BP-lowering effect of patient intervention. Even though physician intervention alone showed limited BP-lowering effects compared with patient intervention alone, this study concluded that combined intervention may have changed the doctor-patient interaction in such a way that the physician was more likely to intensify antihypertensive treatment.

\section{Comments}

Short-term success does not assure long-term benefit. Although this study's primary end point of SBP lowering at 6 months demonstrated the significant BP effects from either patient intervention or combined intervention, Svetkey et al. [3] could not detect long-lasting significant effects on BP at 18 months of either patient intervention alone, physician intervention alone, or synergism of the two interventions. In spite of this disappointing lack of longterm effects on BP, the investigators did observe that effects on behavior (i.e., improved dietary pattern and weight loss) in the combined intervention group at 18 months was comparable to those found at 6 months. This trend is important because the decrease of SBP in the patientintervention group may be attributable to dietary improvement and weight loss [3]. Perhaps effective maintenance of a healthy lifestyle and weight control is the key to extending intervention benefits beyond the 6 months.

The study-design weaknesses in the HIP study have been suggested by Taler [6] in the editorial commentary section of the same journal issue. One caveat concerns a development that arose during the study: most patients already had hypertension controlled at entry (about 60\% were already at goal). Taler suggested that the inclusion of study subjects with BP already at goal may have lessened the motivation for physicians or patients alike to commit to long-term changes that are beneficial to hypertension control, which may explain the relative lack of effects on diet and BP at 18 months. However, that same "limitation" may also be viewed as a strength, in that inclusion of subjects already at BP goal may allow the generalization of study conclusions to a greater fraction of the at-risk population.

Replication is a critical validating step for any scientific result. The HIP study indeed uncovered patterns similar to those observed in two previous reports, the PREMIER trial [7] and a 2006 study by Roumie et al. [8]. Even though the other two trials had differing subject-entry criteria, all three studies showed that physician-only intervention or educa- 
tion provided marginal benefit, whereas patient intervention and education added benefit in BP lowering.

Current hypertension control rates in the United States have been reported in two independent studies to be as low as about $44 \%$ among hypertensive patients $[9,10]$ and only $64 \%$ even among treated hypertensive patients [9]. Because effective control of BP reduces the risk of cardiovascular events [11], there is clearly substantial room for improvement in rates of successful BP control. The HIP study highlights the promise of dual-level, comprehensive, nonpharmacologic BP management strategies. The results of this approach provide physicians with initial evidencebased data upon which clinical practice improvements can be based. The interest of the study resides not only in the importance of the questions asked but also in the significance of the findings on BP.

Certainly, challenges remain in identifying and implementing the most useful and cost-effective strategies to maintain adherence to positive lifestyle changes and treatment compliance even after the initial intervention period has concluded [12]. Other novel interventions that Svetkey et al. [3] have studied include the use of home BPmonitoring devices [13], once again suggesting that combined intervention (home BP monitoring plus tailored behavioral telephone intervention) may be more effective than usual care alone [13]. In conclusion, effective BP management remains an elusive objective for health care providers to achieve. As Svetkey et al. [3] stated, "Future development and testing of both patient and provider interventions should be a high priority" in pursuit of the ultimate goal of successful hypertension treatment.

Disclosure No potential conflict of interest relevant to this article was reported.

Open Access This article is distributed under the terms of the Creative Commons Attribution Noncommercial License which permits any noncommercial use, distribution, and reproduction in any medium, provided the original author(s) and source are credited.

\section{References}

1. Fields LE, Burt VL, Cutler JA, et al.: The burden of adult hypertension in the United States 1999-2000: a rising tide. Hypertension 2004, 44:398-404.

2. Cutler JA, Sorlie PD, Wolz M, et al.: Trends in hypertension prevalence, awareness, treatment, and control rates in United States adults between 1988-1994 and 1999-2004. Hypertension 2008, 52:818-827.

3. Svetkey LP, Pollak KI, Yancy WS Jr, et al.: Hypertension improvement project: randomized trial of quality improvement for physicians and lifestyle modification for patients. Hypertension 2009, 54:1226-1233.

4. Appel LJ, Moore TJ, Obarzanek E, et al.: A clinical trial of the effects of dietary patterns on blood pressure. DASH Collaborative Research Group. N Engl J Med 1997, 336:1117-1124.

5. Chobanian AV, Bakris GL, Black HR, et al.: The Seventh Report of the Joint National Committee on Prevention, Detection, Evaluation, and Treatment of High Blood Pressure: the JNC 7 report. JAMA 2003, 289:2560-2572.

6. Taler SJ: Both the toolset and motivation are needed for lasting change. Hypertension 2009, 54:1202-1203.

7. Appel LJ, Champagne CM, Harsha DW, et al.: Effects of comprehensive lifestyle modification on blood pressure control: main results of the PREMIER clinical trial. JAMA 2003, 289:2083-2093.

8. Roumie CL, Elasy TA, Greevy R, et al.: Improving blood pressure control through provider education, provider alerts, and patient education: a cluster randomized trial. Ann Intern Med 2006, 145:165-175.

9. Ostchega Y, Yoon SS, Hughes J, Louis T: Hypertension awareness, treatment, and control-continued disparities in adults: United States, 2005-2006. NCHS Data Brief 2008, issue $3: 1-8$.

10. Fang J, Alderman MH, Keenan NL, et al.: Hypertension control at physicians' offices in the United States. Am J Hypertens 2008, 21:136-142.

11. Wang JG, Staessen JA, Franklin SS, et al.: Systolic and diastolic blood pressure lowering as determinants of cardiovascular outcome. Hypertension 2005, 45:907-913.

12. Reed SD, Li Y, Oddone EZ, et al.: Economic evaluation of home blood pressure monitoring with or without telephonic behavioral self-management in patients with hypertension. Am J Hypertens 2010, 23:142-148.

13. Bosworth HB, Olsen MK, Grubber JM, et al.: Two selfmanagement interventions to improve hypertension control: a randomized trial. Ann Intern Med 2009, 151:687-695. 